\title{
N-3 fatty acids, neuronal activity and energy metabolism in the brain
}

Emilie HARBEBY ${ }^{1}$

Fabien PIFFERI $^{2}$

Mélanie JOUIN ${ }^{1}$

Hélène PÉLERIN ${ }^{1}$

Sébastien TREMBLAY ${ }^{3,4}$

Roger LECOMTE ${ }^{4}$

Stephen C. CUNNANE ${ }^{3}$

Alain HUERTAS ${ }^{5}$

Jean-Marc ALESSANDRI ${ }^{1}$

Philippe GUESNET ${ }^{6}$

${ }^{1}$ NuRélice,

INRA,

Jouy-en-Josas, France

${ }^{2}$ Mécanismes Adaptatifs et Evolution,

UMR 7179 CNRS,

Muséum National d'Histoire Naturelle,

Brunoy, France

${ }^{3}$ Research Center on Aging and

Department of Medicine,

Université de Sherbrooke,

Sherbrooke, QC, Canada

${ }^{4}$ Departement of Nuclear Medecine

and Radiobiology,

Université de Sherbrooke,

Sherbrooke, QC, Canada

${ }^{5}$ Lesieur,

29 quai Aulagnier,

Asnieres sur Seine,

France

${ }^{6} \mathrm{PG}$ Consulting,

13 villa Bellevue,

Bures sur Yvette,

France

$<$ guesnet07@sfr.fr >

Article received 11 May 2012

Accepted 22 May 2012

\begin{abstract}
The content of docosahexaenoic acid (DHA) in brain membranes is of crucial importance for the optimum development of brain functions. A lack of DHA accretion in the brain is accompanied by deficits in learning behavior linked to impairments in neurotransmission processes, which might result from alteration of brain fuel supply and hence energy metabolism. Experimental data we published support the hypothesis that $n-3$ fatty acids may modulate brain glucose utilization and metabolism. Indeed rats made deficient in DHA by severe depletion of total $n-3$ fatty acid intake have 1) a lower brain glucose utilization, 2) a decrease of the glucose transporter protein content GLUT1 both in endothelial cells and in astrocytes, 3) a repression of GLUT1 gene expression in basal state as well as upon neuronal activation. This could be due to the specific action of DHA on the regulation of GLUT1 expression since rat brain endothelial cells cultured with physiological doses of DHA had an increased GLUT1 protein content and glucose transport when compared to non-supplemented cells. These experimental data highlight the impact of $n-3$ fatty acids on the use of brain glucose, thereby constituting a key factor in the control of synaptic activity. This emerging role suggests that dietary intake of $n-3$ fatty acids can help to reduce the cognitive deficits in the elderly and possibly symptomatic cerebral metabolic alterations in Alzheimer disease by promoting brain glucose metabolism.
\end{abstract}

Key words: Ageing, Alzheimer, Brain, Glucose metabolism, Glucose transporter GLUT1, Docosahexaenoic acid (DHA), N-3 fatty acids

\section{Introduction}

Among nutrients improving human health, $n-3$ polyunsaturated fatty acids ( $n-3$ PUFA) have received wide attention during the last two decades. They support cardiovascular health and optimal infant development and have also promising benefits against several brain and eye diseases (depression, Alzheimer Disease (AD), age-related macular degeneration disease) (Alessandri et al., 2004, 2007; Bazan et al., 2011; Guesnet and Alessandri, 2011). Due to the uniquely high concentration in the membranes of the Central Nervous System (CNS) of the long-chain n-3 PUFA docosahexaenoic acid (DHA, 22:6n-3), n-3 PUFA support fundamental physiological functions in the development and maintain of brain synaptic and retina electrophysiological activities (Alessandri et al., 2004). The molecular mechanisms involved are multiple, directly by regulating dynamic properties of membranes and gene expression and indirectly as precursors of active metabolites such as neuroprotectins (Alessandri et al., 2004; Bazan et al., 2011; Guesnet et al., 2005). In 2002, we acquired experimental data in an animal model of $n-3$ PUFA deficiency which led us to hypothesize that DHA brain concentration could regulate neuronal activity by controlling brain energy metabolism and more specifically glucose utilization and transport (Ximenes et al., 2002). This suggests that beneficial effects of fish and DHA consumption against risk of AD or other forms of dementia, in

To cite this article: Harbeby E, Pifferi F, Jouin M, Pélerin H, Tremblay S, Lecomte R, Cunnane SC, Huertas A, Alessandri JM, Guesnet P. N-3 fatty acids, neuronal activity and energy metabolism in the brain. OCL 2012; 19(4): 238-244. doi : 10.1684/ocl.2012.0459 
which a severe reduction of brain glucose metabolism and cerebral energy metabolism parameters has been reported, could act by this metabolic pathway (Cunnane et al., 2011). The present article aims to provide an update on this field of research and presents possible cellular and molecular mechanisms of n-3 PUFA impact.

\section{N-3 PUFA and brain function}

The involvement of n-3 PUFA in the functional development of the CNS relies on the fact that DHA, the longest and most unsaturated fatty acid of this PUFA family with 22 carbons and 6 double bonds, is the major component of nerve cell membranes in the brain and retina. In the phosphatidylethanolamine (PE) fraction, DHA represents about 25\% (wt\%) of total fatty acids in the human cerebral cortex, about $30 \%$ in the whole retina up to $60 \%$ in the discs of the rod photoreceptor cells. This long-chain PUFA is directly supplied by the diet as marine food products and/or is formed through the metabolic pathway of bioconversion from the essential precursor of the $n-3$ series, alpha-linolenic acid (LNA, 18:3n-3) and eicosapentaenoic acid (EPA, 20:5n-3). However ALA is so insufficiently converted to DHA by the human adult that a dietary intake of preformed DHA is generally recommended (Plourde and Cunnane, 2007).

The essential role of DHA in the maturation of cerebral and visual functions has been mainly demonstrated by the use of animal models specifically diet-deficient in n-3 fatty acids that we (Guesnet et al., 1986) and others have developed. In these nutritional models, animals are fed from conception until the adult age diets based on vegetable oils very rich in n-6 PUFA as linoleic acid and very poor in LNA (Guesnet et al., 1986). The $n-6 / n-3$ imbalance induces a sharp decrease of the DHA membrane concentration in nerve cells from 50 to $80 \%$ (Alessandri et al., 2003) and results in alterations in several behavioural tests, involving learning hability and sensory, motor or motivational processes in the rodent, and impairments in visual acuity and electrical response of the retina in the rodent and the non-human primate (Alessandri et al., 2004). Changes in cognitive performance have been linked to the function of DHA in brain membranes because DHA has been shown to modulate neurogenesis, ion channels, synaptic signaling, activity of transmembrane proteins, and metabolism of several neurotransmitters such as monoamines, acetylcholine and glutamate. Regarding the dopaminergic system, experiments conducted in $n-3$ deficient rats demonstrated that several parameters of neurotransmission were affected at the same time in the frontal cortex (Chalon et al., 2006). Notably data from microdialysis technique have suggested an alteration of the vesicular dopamine pool and its release from presynaptic vesicles. Such impairments can be also observed in a nutritional state of moderate n-3 PUFA deficiency. In a dose-response study in which the brain DHA concentration varied from $25 \%$ to $120 \%$ of the control value by increasing the dietary DHA supply, it was pointed out that a brain DHA content of $70 \%$ of the control value was not sufficient to normalize the dopamine pool. This therefore suggests that brain membranes require a high DHA content for their optimal functioning (PoumèsBallihaut, 2002). Neurophysiological effects of DHA involve an extraordinary diversity of molecular mechanisms and regulation pathways. Among them, the membrane structure and dynamics (phospholipid composition and lipid raft formation), the synaptic signaling involving eicosanoids and docosanoids, and the regulation of gene expression in the brain by nuclear factors are the most important (Alessandri et al., 2004).

Dietary n-3 deficiency has been also reported in humans, primarily in newborn infants who received infant formula low in total n-3 PUFA. Indeed clinical studies have shown that feeding term or premature infants with formula low in LNA and devoid of DHA delayed development of visual acuity (Guesnet and Alessandri, 2011).

\section{Neuronal activity and brain glucose utilization and metabolism}

Although the brain represents only $2 \%$ of the body mass, it consumes about $25 \%$ of glucose and $20 \%$ oxygen used by the body. Glucose is the principle energy fuel of the brain which is consumed in human at about $100 \mathrm{~g} / \mathrm{d}$. In special nutritional situations (fasting, high fat/low carbohydrate diet) or illness, glucose could be partially replaced by alternative energy substrates as ketone bodies. Most of the energy used by the brain is required to ensure the process of neurotransmission, mostly for restoring action potentials and postsynaptic currents (Attwell and ladecola, 2002) (figure 1). Notably the reversion of $\mathrm{Na}+$ and $\mathrm{K}+$ fluxes across the cell membrane consumes about $80 \%$ of brain ATP by requiring the activation of the neuronal $\mathrm{Na}+/ \mathrm{K}+$ ATPase coupled to the inversion of ion fluxes against their concentration gradient. Moreover, the functioning of the glutamatergic synapse and the glutamate uptake by astrocytes for glutamate recycling also requires the activation of a $\mathrm{Na}+/ \mathrm{K}+$ ATPase pump (astrocytic form) and the use of ATP (figure 1).

Neuronal activity is tightly coupled to glucose utilization and thus with the increased transfer of glucose from the blood to neurons and astrocytes. Glucose is distributed from the blood to the brain cells via specific membrane glucose transporters (GLUTs), mainly GLUT1 and GLUT3. It is first transported across the endothelial cells of the blood-brain barrier (BBB) by the 55-kDa GLUT1 localized at the luminal (facing the blood) and abluminal (facing the parenchyma) sides of the cells, then into astrocytes by the 45-kDa GLUT1 isoform and into neurons by GLUT3 (Duelli and Kuschinsky, 2001) (figure 1). In the basal state, GLUT1 and GLUT3 are the key regulators of brain glucose consumption since their density in brain areas consistently parallels local rates of glucose utilization (Duelli et al., 2001). During neuronal activation, the local brain glucose utilization is increased in response to the increase of ATP consumption linked to the activation of neuronal and astrocytic $\mathrm{Na}+/ \mathrm{K}+$ ATPases. To support brain glucose utilization, adaptive changes occur simultaneously such as increases of local cerebral blood flow and of oxygen utilization. GLUT1 appears here to be the main factor responding to rapid changes in the increased glucose demand. Indeed, the activation of a brain area involved in memory processing, the hippocampus, specifically increases GLUT1 gene and protein expressions of both endothelial and astrocytic isoforms (Choeiri et al., 2005). On the other side, the expression of neuronal GLUT3 does not change, supporting the concept 


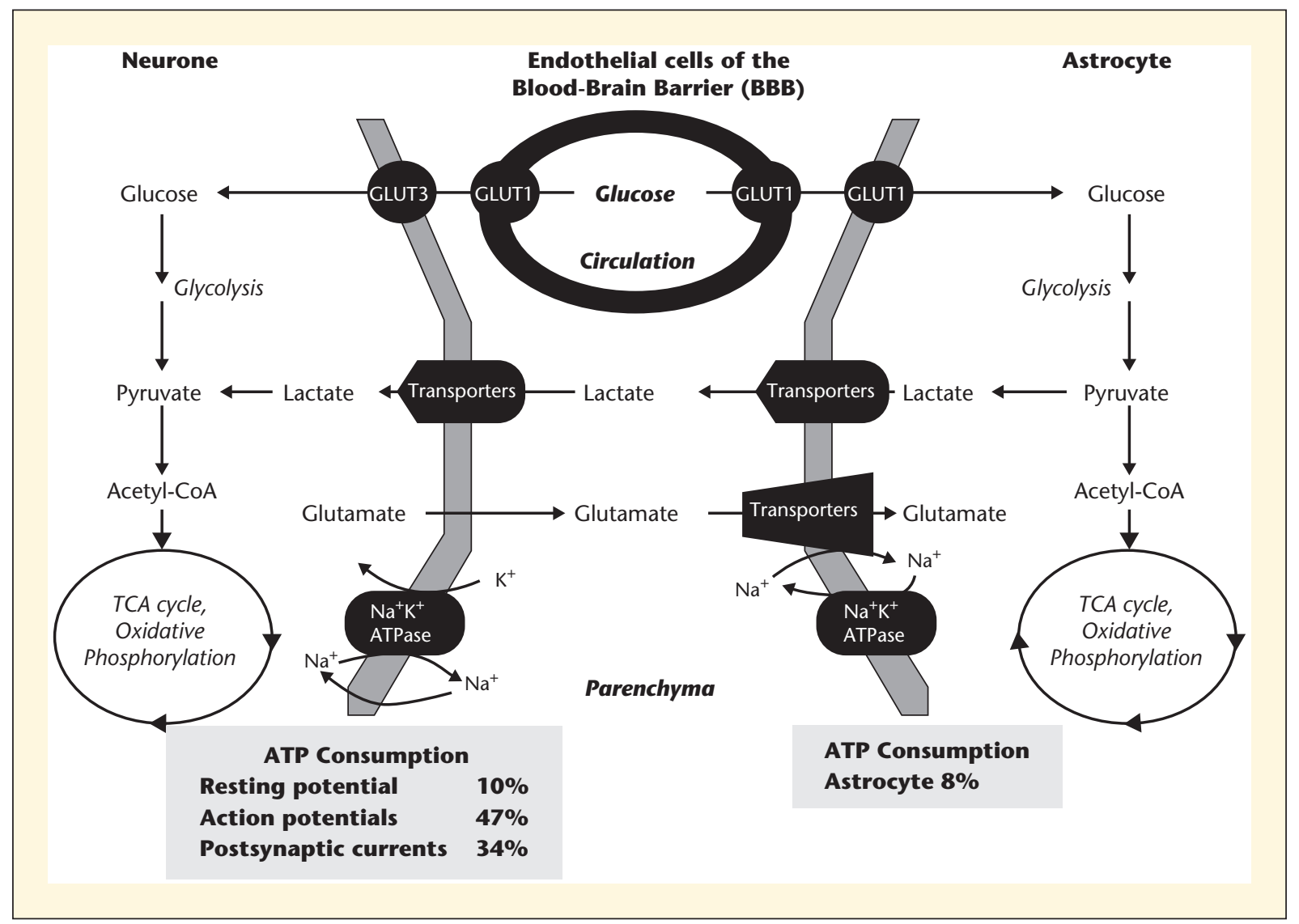

Figure 1. Neuroenergetics and synaptic activity: the glutamatergic synapse. Whatever the type of synapses, most of the brain energy in the rodent is used for reversing $\mathrm{Na}+$ and $\mathrm{K}+$ fluxes provoked by action potentials (47\% of brain ATP consumed) and postsynaptic currents (34\%). The reversion of ion fluxes is mediated by the neuronal $\mathrm{Na}+\mathrm{K}+$ ATPase. About $10 \%$ of brain ATP is expended at the level of the glutamatergic synapse for the recycling of glutamate released in the synaptic left since it is coupled to $\mathrm{Na+}$ influx and astrocytic $\mathrm{Na+} / \mathrm{K}+$ ATPase activation. Glucose, the main energy substrate, is translocated from blood via endothelial cells of the blood-brain barrier (BBB) by a glucose transporter GLUT1 (55 kDa isoform), and is then taken up by astrocytes via GLUT1 (45 kDa isoform) and by neurons via GLUT3. In astrocytes, part of the pyruvate produced from glucose is converted to lactate which is an alternative energy substrate for neurones during neuronal activation.

that neurons are metabolically unable to increase their glucose uptake and utilization upon activation to sustain ATP demand (Choeiri et al., 2005). We reported similar data for GLUT gene expression in the fronto-parietal cortex of rats upon neuronal activation after exposition to an enriched environment (Harbeby et al., 2012).

In laboratory animals but also in the human, regional brain activity may be monitored by the measurement of local rate of glucose utilization with the functional imaging technique positron emission tomography (PET). The tracer of choice used is the glucose analogue 2-deoxyglucose (2DG) which is transported into the brain at the same rate as glucose. However, 2DG cannot be metabolized further the first step of glycolysis and therefore accumulates intracellularly, thus representing the amount of glucose utilized. A positronemitting radionuclide, the fluorine-18 (18F), is incorporated as tracer into 2DG and is then quantified by a $\gamma$-detector. PET scan has been widely used for the measurement of the rate of brain glucose uptake in aged and Alzheimer's patients (Cunnane et al., 2011).

\section{N-3 PUFA and brain cerebral glucose metabolism}

Data from rat studies have shown that n-3 PUFA deficiency decreases DHA in brain membranes and alters monoaminergic and glutamatergic synaptic function, leading to deficits in learning and memory behavior. We hypothesized that such alterations in synaptic functions, which use most of the brain energy as described above, could result from changes in brain energy metabolism and possibly from glucose hypometabolism. A previous study supported this hypothesis, reporting that the activity of the $\mathrm{Na}+\mathrm{K}+$ ATPase pump was severely reduced $(-50 \%)$ in nerve endings in the whole brain of $n-3$ PUFA deficient rat (Bourre et al., 1989). Therefore, a first study was conducted in the rat to determine whether the brain glucose metabolism was functionally altered in a situation of nutritional deficiency. The rate of cerebral glucose utilization was measured using the semi-quantitative autoradiographic 2DG method (Ximenes et al., 2002). Experimental data clearly pointed out a global brain glucose hypometabolism 
induced by $\mathrm{n}-3$ deficient because in comparison to control rats receiving an equilibrated PUFA diet, a significant decrease (about - 30\%) in the rate of glucose utilization was noted in several brain regions and specifically in the cerebral cortex (fronto-parietal region) (figure 2 Part A). This lower rate of brain glucose utilization was confirmed for the whole brain in a recent FDG-dynamic PET study. A significant reduction of 18F-FDG uptake was evidenced in the whole brain of these animals resulting to both a lower rate of brain uptake during the early phase of the kinetic (0-15 min) and a lower plateau level of brain incorporation during the later plateau phase (15-45 min) (figure 2 Part A) (Harbeby et al., 2010). In contrast, no alteration in the blood glucose concentration nor in the arterial blood pressure was reported.

Since total GLUT1 protein immunocytochemical staining was significantly lower in the cerebral cortex of deficient rats (figure 2 Part B) and that GLUT1 transporter is a key regulator of brain glucose consumption, we postulated that the main cause of alteration in glucose metabolism was a functionally abnormal GLUT1 protein. Decrease GLUT1 protein expression was then confirmed for the 2 GLUT1 isoforms by Western blot quantification on cortical isolated microvessels (55-kDa endothelial isoform) and homogenate (45-kDa astrocytic isoform) (Pifferi et al., 2005). Our data clearly indicate that $n-3$ PUFA deficiency specifically decreased the GLUT1 protein content of both endothelial cells and astrocytes by $25-30 \%$ (figure 2 Part B). Determination of cytochalasin B binding on the cortical brain microvessels, a measure of the density of endothelial GLUT1, confirmed that GLUT1 protein density was decreased at the BBB level in n-3 deficient rat (figure 2 Part B) (Pifferi et al., 2007). The lower protein amount of GLUT1 transporters results from a 25 to $30 \%$ reduction of the transcription of the gene encoding the two GLUT1 isoforms (Slc2a1) (figure 2 Part B) (Harbeby et al., 2012). This reduction in gene expression was also found during neuronal activation, supporting the hypothesis that the alteration of glucose uptake due to $\mathrm{n}-3$ deficiency persists during brain activation (Harbeby et al., 2012). A similar reduction of GLUT1 gene expression was also found in the olfactory bulb and the neocortex of $\mathrm{n}-3$ deficient rats placed in basal and in olfactory learning conditions (Hichami et al., 2007).

As a whole, experimental data acquired in $n-3$ deficient rats demonstrated clearly that dietary intake of $n-3$ fatty acids modulates, via DHA in membranes, brain glucose uptake. This involves the regulation of the key step controlling the delivery of blood glucose to brain cells for sustaining glucose utilization and neuronal activity i.e. GLUT1 at the BBB level. Data also suggest that the transport and metabolism of glucose could be also altered upon neuronal activation, possibly at the level of lactate production by astrocytes. The specificity of $n-3$ effect is highlighted by the absence of effect on neuronal GLUT3 glucose transporter (figure 2 Part B). All the data are reinforced by a recent Primate study which pointed out that regional differences in brain DHA concentration are directly proportional to brain glucose uptake in the same regions (Brenna and Diau, 2007).

\section{Mechanisms of action of n-3 PUFA}

Rats deficient in n-3 PUFA have decreased brain glucose utilization which is associated with reduced protein and gene expression of the endothelial and astrocytic GLUT1. These effects were ascribed to changes in PUFA composition in brain membranes including those of microvessels, with subnormal DHA content and high n-6 PUFA content. Since oxidative phosphorylation activity was also impaired by the deficiency (Ximenes et al., 2002), reduced glucose transport may result from a direct effect of lower DHA content reducing the activity of endothelial GLUT1, or may be a secondary consequence of the depressed oxidative phosphorylation. To test the hypothesis that DHA in brain endothelial cell membranes could directly modulate glucose uptake and GLUT1, we studied the responses of primary cultures of rat brain endothelial cells (RBEC) in terms of glucose uptake and GLUT1 expression, to supplements of DHA in comparison to the long-chain n-6 PUFA arachidonic acid (AA, 20:4n-6). First it must be emphasized that RBEC cultured without PUFA supplementation (basal condition) have very low DHA content in their membrane phospholipids, which is equivalent to that found in microvessels of rats fed a n-3 deficiency diet (Pifferi et al., 2007). DHA added to the culture medium at a dose of $15 \mu \mathrm{M}$ restored the natural DHA content of brain endothelial cells, and concurrently increased the basal uptake of glucose and the GLUT1 protein expression compared to control cultures (figure 3) (Pifferi et al., 2010). The stimulatory effect was specific for long-chain n-3 PUFA since eicosapentaenoic acid (20:5n-3) produced very similar effects whereas AA had no impact on these parameters. Preliminary in vitro studies on astrocytes suggest that $n-3$ PUFA can also alter glucose uptake in glial cells by modulating the synthesis of GLUT1 protein (Guesnet et al., unpublished results).

Molecular mechanisms of DHA action could implement both transcriptional, post-transcriptional (transcript stability) and post-translational events of GLUT1 expression (Duelli and Kuschinsky, 2001). DHA may modulate gene transcription through the activation of peroxisome proliferator-activated receptors (PPARs) because it is a potent endogenous ligand for these transcriptional factors in neural cells (Niemoller and Bazan, 2010).

Alteration of cerebral glucose uptake in n-3 fatty acid deficient animals could also be explained as a consequence of the depressed mitochondrial oxidative phosphorylation (OXPHOS) pathway and possibly of ATP production. A significant decrease in the activity of mitochondrial cytochrome $\mathrm{C}$ oxidase activity was reported in several brain regions in rats deficient in $\mathrm{n}-3$ fatty acids (Ximenes et al., 2002). Additionally, the gene expression of several OXPHOS genes encoding subunits of complexes involved in electron transport chain and of the critical enzyme F1F0 ATP synthase was affected by variation of dietary n-3 PUFAs (Kitajka et al., 2004; Harbeby et al., 2012).

\section{Conclusions - Potential interest of $\mathbf{n}-\mathbf{3}$ PUFA for ageing brain}

Data from animal studies suggest that DHA may be an important factor for normal brain energy metabolism and glucose utilization by regulating the key step controlling the delivery of blood glucose to brain cells, the glucose transporter GLUT1. A pilot PET study on patients with depressive symptoms supports this hypothesis by demonstrating a positive 


\section{A- Brain glucose utilization}

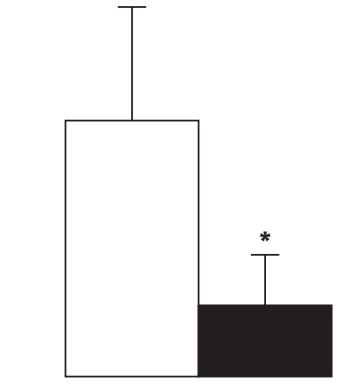

Rate of uptake

$\left[1-^{14} \mathrm{C}\right] 2 \mathrm{DG}$ study

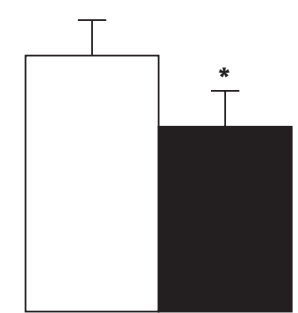

Rate of uptake

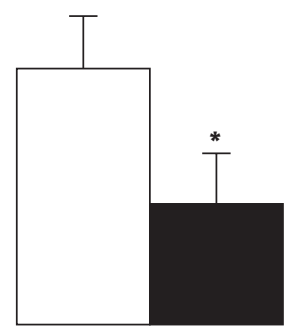

Rate in early phase

\section{FDG-PET study}

\section{B- Brain GLUT expression}

GLUT1

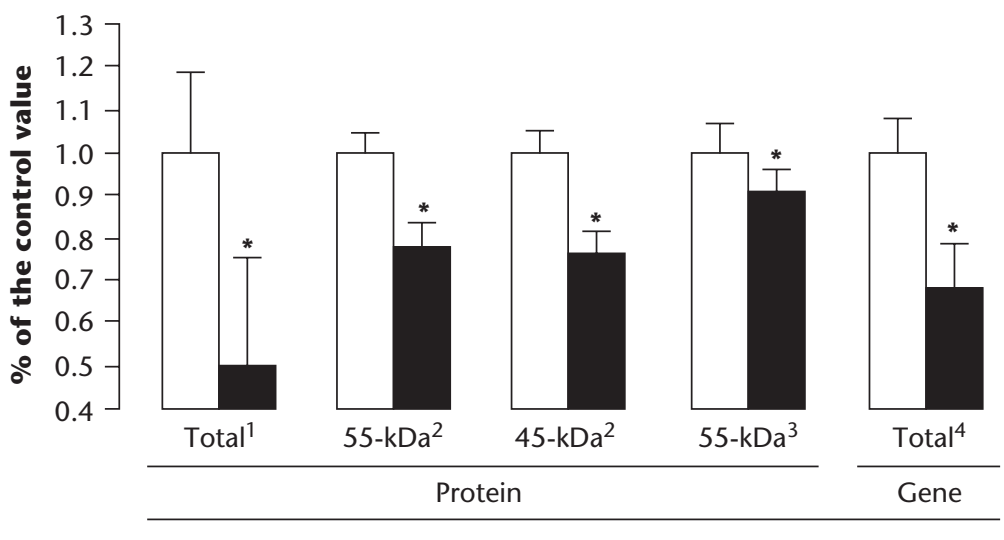

Control

N-3 Deficient

(1)

-

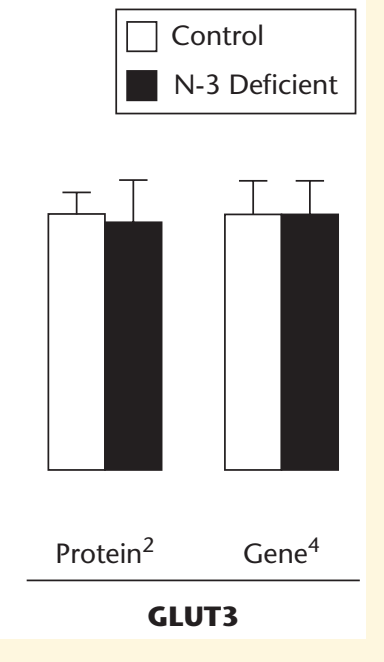

Figure 2. Impact of n-3 PUFA deficiency on glucose utilization (Part A) and GLUT expression (Part B) in the rat brain at rest. Part A. Cerebral glucose utilization was evaluated in the frontal cortex using the semiquantitative autoradiographic [1-14C] 2-deoxyglucose (2DG) method (Ximenes et al., 2002), and in the whole brain using the [18F]-2DG positron emission tomography (FDG-PET) (Harbeby, 2011). The FDG uptake was calculated both during the later plateau and the early phases of the kinetic. Part B. ${ }^{1}$ The level of total GLUT1 staining was measured in the fronto-parietal cortex by immunocytochemistry on $30 \mu \mathrm{m}$ coronal sections (Ximenes et al., 2002). ${ }^{2}$ GLUT1 and GLUT3 protein levels were assayed by western blotting on samples of cerebral cortex homogenates (GLUT1 45-kDa and GLUT3) and microvessels (GLUT1 55-kDa) (Pifferi et al., 2005). ${ }^{3}$ Quantification of GLUT1 55 kDa was also performed by determining the specific binding of cytochalasin B to GLUT1 on total membranes prepared from isolated microvessels (Pifferi et al., 2007). ${ }^{4}$ Level of mRNA expression of GLUT1 gene (Slc2a1) determined by realtime PCR TaqMan Low-Density Array (TLDA) technique (Harbeby et al., 2012). Bars represent means \pm SEM ( $n=6-8$ rats/diet group). * Statistically different from the control rats $(P<0.05)$.

correlation between plasma DHA and glucose metabolic rates in the frontal and temporo-parietal cortices (Sublette et al., 2009). This indicates that individuals with lower $\mathrm{DHA}$ levels had relative glucose hypometabolism in this region.

Therefore, it is tempting to propose the hypothesis that n-3 fatty dietary intake might exert protective effects against risk of Alzheimers disease (AD) or other forms of dementia and possibly neuropathologies by correcting, among other benefi- cial effects, the alterations of brain energy and glucose metabolism. Cunnane et al. (2011) have recently reviewed the published evidence suggesting that glucose hypometabolism in hippocampus and several cortical regions is a characteristic of AD. It is associated with low activity of glycolytic enzymes and mitochondrial dysfunction. These alterations can be observed present in advance of the onset of measurable cognitive decline and also has been noted in some studies of normal aging. Prospective studies showed that high dietary intake of fish or long-chain n-3 PUFA (mainly DHA) 


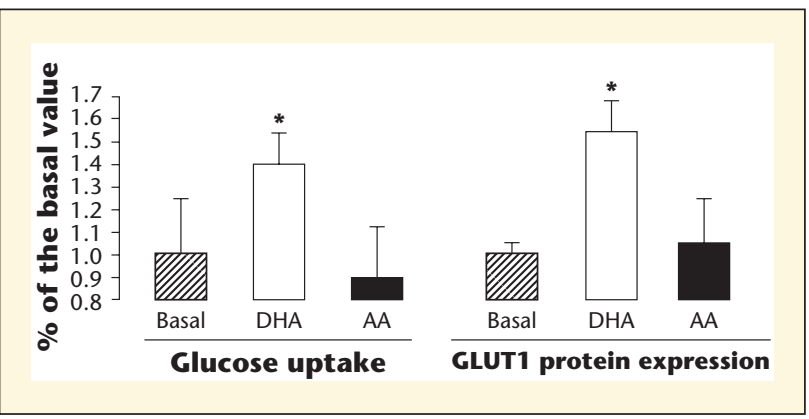

Figure 3. Impact of DHA supplementation on glucose uptake in rat brain endothelial cells primary culture (RBEC). Glucose uptake ([3H]3-o-methyl glucose method) and GLUT1 protein expression (Western blotting) were measured in RBEC cultived in basal condition (no PUFA supplementation) or supplemented with $15 \mu \mathrm{M}$ docosahexaenoic acid $(D H A, 22: 6 n-3)$ or arachidonic acid ( $A A, 20: 4 n$-6) (Pifferi et al., 2010). Glucose uptake was measured using the non-metabolisable glucose analogue 3-o-methylglucose. Values are means $\pm S D(n=4$ separate experiments each performed in triplicate). *Statistically different from non-supplemented cells (basal) $(P<0.05)$.

could contribute significantly to the reduction of the risk of $A D$ and of cognitive decline, and animal studies have reported protective effect of dietary DHA upon cognitive alterations in ageing and AD models. Therefore it is tempting to propose the hypothesis that dietary intake of $n-3$ PUFA might exert protective effects against risk of $A D$ or other forms of dementia by modulating brain energy and glucose metabolism.

Acknowledgments. Dr. Philippe Guesnet wishes to dedicate this presentation to Jean Klere who died in 2011 and was awarded the Médaille Chevreul in 1986 in the field of margarine technology. He thanks him for introducing him to the field of lipids, technology, and application. Dr Philippe Guesnet particularly thanks the company Lesieur which funded part of this fundamental work with efficiency and enthusiasm. This work was also supported by l'Institut National de la recherche Agronomique (INRA), le Groupe Lipides Nutrition (GLN), I'Agence National de la Recherche (ANR, PNRA program "Nutrisens"), and Dr Philippe Guesnet. The PhD Thesis of Dr Emilie Harbeby was co-financed by INRA and the company Lesieur, and the PhD Thesis of Dr Fabien Pifferi was supported by grant from the French Ministère de I'Éducation Nationale de la Recherche et de la Technologie. S. Cunnane was funded by NSERC Canada.

\section{REFERENCES}

Alessandri JM, Poumès-Ballihaut $C$, Langelier $B$, et al. Incorporation of docosahexaenoic acid into nerve membrane phospholipids: bridging the gap between animals and cultured cells. Am / Clin Nutr 2003; 78: 702-10.

Alessandri JM, Guesnet P, Vancassel S, et al. Polyunsaturated fatty acids in the central nervous system: evolution of concepts and nutritional implications throughout life. Reprod Nutr Dev 2004; 44: 509-38.

Alessandri JM, Astorg P, Chardigny JM, et al. Acides gras polyinsaturés (AGPI) - Structure, métabolisme, fonctions biologiques, apports nutritionnels recommandés et principales sources alimentaires. In: M.
Robertfroid, V. Coxam, N. Delzenne (Eds.), Aliments Fonctionnels, $2^{\mathrm{e}}$ Edition, Paris: Ed Tec \& Doc-Lavoisier, 2007: 161-96.

Attwell $D$, ladecola $C$. The neural basis of functional brain imaging signals. Trends Neurosci 2002; 25: 621-5.

Bazan NG, Molina MF, Gordon WC. Docosahexaenoic acid signalolipidomics in nutrition: significance in aging, neuroinflammation, macular degeneration, Alzheimer's, and other neurodegenerative diseases. Annu Rev Nutr 2011; 21: 321-51.

Bourre JM, Francois M, Youyou A, Dumont O, Piciotti M, Pascal G, Durand G. The effects of dietary alpha-linolenic acid on the composition of nerve membranes, enzymatic activity, amplitude of electrophysiological parameters, resistance to poisons and performance of learning tasks in rats. J Nutr 1989; 119: 1880-92.

Brenna JT, Diau GY. The influence of dietary docosahexaenoic acid and arachidonic acid on central nervous system polyunsaturated fatty acid composition. Prostaglandins Leukot Essent Fatty Acids 2007; 77: 247-50.

Chalon S. Omega-3 fatty acids and monoamine neurotransmission. Prostaglandins Leukot Essent Fatty Acids 2006; 7: 259-69.

Choeiri C, Staines W, Miki T, Seino S, Messier C. Glucose transporter plasticity during memory processing. Neuroscience 2005; 130: 591 600.

Cunnane SC, Nugent S, Roy M, et al. Brain fuel metabolism, aging, and Alzheimer's disease. Nutrition 2011; 27: 3-20.

Duelli R, Kuschinsky W. Brain glucose transporters: relationship o local energy demand. News Physiol Sci 2001; 16: 71-6.

Duelli R, Maurer MH, Staudt R, Sokoloff L, Kuschinsky W. Correlation between local glucose transporter densities and local 3-O-methylglucose transport in rat brain. Neurosci Lett 2001; 310: 101-4.

Guesnet P, Pascal G, Durand G. Dietary alpha-linolenic acid deficiency in the rat. I. Effects on reproduction and postnatal growth. Reprod Nutr Dev 1986; 26: 969-85.

Guesnet P, Alessandri JM, Astorg P, Pifferi F, Lavialle M. Les rôles physiologiques majeurs exercés par les acides gras polyinsaturés (AGPI). OCL 2005; 12: 333-43.

Guesnet P, Alessandri JM. Docosahexaenoic acid (DHA) and the developing central nervous system (CNS) - Implications for dietary recommendations. Biochimie 2011; 93: 7-12.

Harbeby E, Tremblay S, Mercier-Tremblay J, et al. Omega- 3 fatty acids and brain glucose utilization: an 18F-fluorodeoxyglucose positron emission tomography (FDG-PET) study in the rat. Poster. ISSFAL 2010, Maastricht, Netherlands. P 161.

Harbeby E, Jouin M, Alessandri JM, et al. N-3 PUFA status affects expression of genes involved in neuroenergetics differently in the fronto-parietal cortex compared to the CA1 area of the hippocampus: Effect of rest and neuronal activation in the rat. Prostaglandins Leukot Essent Fatty Acids 2012 (in Press).

Hichami A, Datiche F, Ullah S, Liénard F, Chardigny JM, Cattarelli M, Khan NA. Olfactory discrimination ability and brain expression of cfos, Gir and Glut1 mRNA are altered in n-3 fatty acid-depleted rats. Behav Brain Res 2007; 184: 1-10.

Kitajka K, Sinclair AJ, Weisinger RS, Weisinger HS, Mathai M, Halver JE, Puskás LG. Effects of dietary omega-3 polyunsaturated fatty gene expression. Proc Natl Acad Sci USA 2004; 101: 10931-6.

Niemoller TD, Bazan NG. Docosahexaenoic acid neurolipidomics, Prostaglandins Other Lipid Mediat 2010; 91: 85-9.

Pifferi F, Roux F, Langelier B, Vancassel S, Jouin M, Lavialle M, Guesnet P. (n-3) polyunsaturated fatty acid deficiency reduces the expression of both isoforms of the brain glucose transporter GLUT1 in rats. J Nutr 2005; 135: 2241-6. 
Pifferi F, Jouin M, Alessandri JM, et al. n-3 Fatty acids modulate brain glucose transport in endothelial cells of the blood-brain barrier. Prostaglandins Leukot Essent Fatty Acids 2007; 77: 279-86.

Pifferi F, Jouin M, Alessandri JM, et al. n-3 long-chain fatty acids and regulation of glucose transport in two models of rat brain endothelial cells. Neurochem Int 2010; 56: 703-10.

Plourde M, Cunnane SC. Extremely limited synthesis of long chain polyunsaturates in adults: implications for their dietary essentiality and use as supplements. Appl Physiol Nutr Metab 2007; 32: 619-34.

Poumès-Ballihaut $C$. Effets d'un apport alimentaire en acide docosahexaénoïque (DHA, 22: 6n-3) sur la composition lipidique membranaire du cerveau et de la rétine, et sur 2 fonctions neurophysiologiques chez le rat: la neurotransmission dopaminergique corticale et la réponse électrique de la rétine. Doctorat de l'Ecole Nationale Supérieure Agronomique de Rennes 2002, pp263.

Sublette ME, Milak MS, Hibbeln JR, et al. Plasma polyunsaturated fatty acids and regional cerebral glucose metabolism in major depression. Prostaglandins Leukot Essent Fatty Acids 2009; 80: 57-64.

Ximenes da Silva A, Lavialle F, Gendrot G, Guesnet P, Alessandri JM, Lavialle M. Glucose transport and utilization are altered in the brain of rats deficient in n-3 polyunsaturated fatty acids. I Neurochem 2002; 81: 1328-37. 